\title{
Sex-specific programming of hypertension in offspring of late-gestation diabetic rats
}

\author{
Ragheed Katkhuda', Emily S. Peterson' ${ }^{1}$, Robert D. Roghair', Andrew W. Norris' ${ }^{1}$, Thomas D. Scholz' and Jeffrey L. Segar ${ }^{1}$
}

BACKGROUND: The intrauterine environment strongly influences adult disease susceptibility. We used a rat model of thirdtrimester maternal diabetes to test the hypothesis that adult offspring exposed to hyperglycemia in utero display increased blood pressure and alterations in vascular responsiveness.

METHODS: Diabetes was induced by streptozotocin injection to pregnant rats on gestation day 13 (term $21 \mathrm{~d}$ ) and partially controlled with insulin injections. Hemodynamic function was evaluated in 6-12-mo-old offspring.

RESULTS: Male but not female offspring of diabetic mothers (ODM) had significantly increased blood pressure as compared with controls; heart rate (HR) was similar. For both sexes, HR baroreflex responses were similar as were in vivo hemodynamic responses to angiotensin II, nitric oxide synthase inhibition, and ganglionic blockade. Aortic contractility to angiotensin II was similar in the two groups. Nitric oxide synthase inhibition and the $\mathrm{Cu} / \mathrm{Zn}$ superoxide dismutase inhibitor diethyldithiocarbamate, but not the superoxide dismutase-mimetic Tempol, significantly increased contractile responses to angiotensin II in controls but not ODM. Reduced nicotinamide adenine dinucleotide phosphate-stimulated superoxide production was greater in male ODM than in controls $(P<0.05)$.

CONCLUSION: Exposure to hyperglycemia in utero results in sex-specific cardiovascular changes in adult offspring. Impaired nitric oxide-reactive oxygen species signaling may play a significant role in the hemodynamic phenotype of ODM.

$\mathbf{T}$ he number of pregnancies complicated by diabetes mellitus continues to increase throughout the developed world, a figure that will rise with the increasing prevalence of type 2 diabetes among young adult women $(1,2)$. Infants of diabetic mothers represent a group of patients with an increased risk of perinatal mortality and morbidity, including cardiomyopathy, macrosomia, hypoglycemia, hypocalcemia, and respiratory distress syndrome (3). Recently, long-term complications in offspring of diabetic mothers have been recognized, including development of type 2 diabetes, dyslipidemia, and obesity independently of the genetic background of the offspring (4-8). Although considerable attention is focused on the increased metabolic abnormalities in adult offspring of diabetic mothers, cardiovascular alterations, including hypertension, are also lifelong consequences of in utero exposure to maternal diabetes. In a Pima Indian population, offspring of mothers who suffered diabetes during pregnancy had higher systolic arterial blood pressure in adolescence as compared with offspring of mothers who developed type 2 diabetes mellitus after the index pregnancy, independent of obesity (9). In a separate population enrolled in the Diabetes in Pregnancy follow-up study, 10-14-y-old offspring of diabetic mothers (ODM) had significantly higher systolic and mean arterial blood pressures (MABPs) than offspring of nondiabetic mothers (10).

The etiology of hypertension in ODM has not been extensively explored. The majority of studies examining cardiovascular dysfunction in ODM have used animals made diabetic either before pregnancy or early in pregnancy by injection of streptozotocin (STZ), an alkylating agent with selective toxicity to the beta cells of pancreatic islets (11). Studies in rats demonstrate that male but not female offspring of dams made diabetic before mating or on the 7 th day of pregnancy are hypertensive by $2-3$ mo of age (12-14). Findings of these studies and others suggest changes in baroreflex sensitivity, vascular responsiveness, activity of the renin-angiotensin and nitric oxide (NO) systems, and arachidonic acid metabolism may contribute to the development of hypertension in ODM $(15,16)$. Because gestational-onset diabetes, characterized by maternal hyperglycemia during the last third of gestation, is the most common form of diabetes to affect pregnancy, our laboratory has focused on a rat model whereby maternal hyperglycemia is induced by STZ at the beginning of the third and final week of gestation. Using this model of maternal diabetes, with hyperglycemia moderated by twice daily insulin, we previously described significantly enhanced aortic contractility and endothelial dysfunction in female but not male offspring (17). We therefore sought to extend studies in this model to determine whether offspring exposed to short-term (7-day) maternal hyperglycemia in utero would display sex-specific increased blood pressure. As studies in animal models suggest abnormalities in autonomic function may precede overt hypertension, we also analyzed baroreflex control of heart rate (HR) (18). Finally, we chose to further our previously reported findings of altered ex vivo aortic vascular responsiveness and endothelial dysfunction in offspring of hyperglycemic mothers

The first two authors contributed equally to this work.

'Department of Pediatrics, University of lowa Carver College of Medicine, lowa City, lowa. Correspondence: Jeffrey L. Segar (jeffrey-segar@uiowa.edu) 
and explore potential mechanisms contributing to our previous observations. Diabetes during pregnancy is associated with increased markers of oxidative injury in the offspring $(19,20)$. Therefore, the isolated vessels studies focused on the contribution of reactive oxygen species to ex vivo aortic vascular dysfunction. Of note, we cross-fostered all pups to nondiabetic mothers to ensure that any identified pathophysiology would be related to the in utero environment and not alterations in postnatal nutritional intake or behavioral patterns associated with maternal diabetes.

\section{RESULTS}

\section{In Vivo Experiments}

Male ODM displayed significantly greater resting mean arterial pressure (123 \pm 5 vs. $102 \pm 4 \mathrm{~mm} \mathrm{Hg} ; P<0.01)$ but similar resting HR ( $360 \pm 11$ vs. $370 \pm 7 \mathrm{bpm})$ as compared with controls (Figure 1). In contrast, no differences in MABP or HR were identified between female ODM and control animals. Peak changes in MABP and HR in response to phenylephrine and nitroprusside were similar in male ODM and control animals (Figure 2). Female ODM also had similar increases in MABP and reflex bradycardia as compared with controls in response to phenylephrine (Figure 2). However, female ODM demonstrated greater reflex tachycardia in response to sodium nitroprusside $(10,20$, and $40 \mu \mathrm{g} / \mathrm{kg})$ as compared with controls, although MABP responses were similar between groups (Figure 2). To evaluate baroreflex sensitivity, slopes of the linear regression line relating percentage change from baseline for mean blood pressure and HR in response to phenylephrine and sodium nitroprusside for each animal were calculated. No differences in the MABP-HR relationship were identified between ODM and control animals for either male $(-1.1 \pm 0.14$ vs. $-1.01 \pm 0.18 \% \triangle \mathrm{HR} / \% \triangle \mathrm{MABP}$, respectively) or female animals $(-1.56 \pm 0.24$ vs. $-1.2 \pm 0.22 \% \Delta \mathrm{HR} / \% \triangle \mathrm{MABP}$, respectively; $P=0.0548)$.

Changes in MABP and HR in response to the NO synthase inhibitor $\mathrm{N}^{\mathrm{G}}$-nitro-L-arginine methyl ester (L-NAME) and the ganglionic blocking agent chlorisondamine are shown in Figure 3. No differences in hemodynamic responses to either agent were seen between ODM and control animals of either sex. Changes in MABP and HR in response to angiotensin II alone or in the presence of L-NAME were also similar between groups in both sexes (Figure 4).

\section{Ex Vivo Vascular Function in Male ODM}

Aortic segments from male ODM and control animals exhibited similar vasoconstrictive responses to angiotensin II (Figure 5). However, in the presence of the angiotensin II type 2 receptor antagonist PD123319, the response to angiotensin II was attenuated in ODM as compared with controls (Figure 5a). Constrictive responses to angiotensin II in the presence of the angiotensin II type 1 receptor antagonist losartan were completely blocked in both groups (data not shown). The $\mathrm{NO}$ synthase inhibitor $\mathrm{N}^{\mathrm{G}}$-nitro-L-arginine (L-NNA) and the $\mathrm{Cu} / \mathrm{Zn}$ superoxide dismutase inhibitor diethyldithiocarbamate (DETC) significantly increased responses to angiotensin II in controls but not ODM, whereas the superoxide scavenger Tempol attenuated responses to angiotensin II in controls but not ODM (Figure 5b-d). The combined effects of L-NNA with DETC resulted in similar changes to either L-NNA or DETC, without any evidence of additive effects (Figure 5e). Similarly, combined L-NNA and Tempol resulted is changes in responses similar to L-NNA alone in both groups (Figure $5 \mathrm{f}$ ).

\section{Ex Vivo Vascular Function in Female ODM}

Similar to that seen in males, aortic segments from female ODM and control animals exhibited similar vasoconstrictive responses to angiotensin II alone (Figure 6a). No effect on constrictive responses was seen in the presence of PD 123319 (Figure 6a). L-NNA and DETC again significantly increased responses to angiotensin II in controls but not ODM, whereas Tempol had no significant effect on the response (Figure $\mathbf{6 b}-\mathbf{d}$ ). As seen in males, the combined effects of L-NNA with DETC resulted in similar changes to either L-NNA or DETC, without any evidence of additive effects (Figure 6e). Of note, the response to angiotensin II in the presence of Tempol + L-NNA was similar between control and ODM female groups (Figure 6f).

\section{Superoxide Production}

Basal superoxide production, as determined by chemiluminescence was similar between groups in both sexes. Reduced nicotinamide adenine dinucleotide phosphate (NADPH)-stimulated
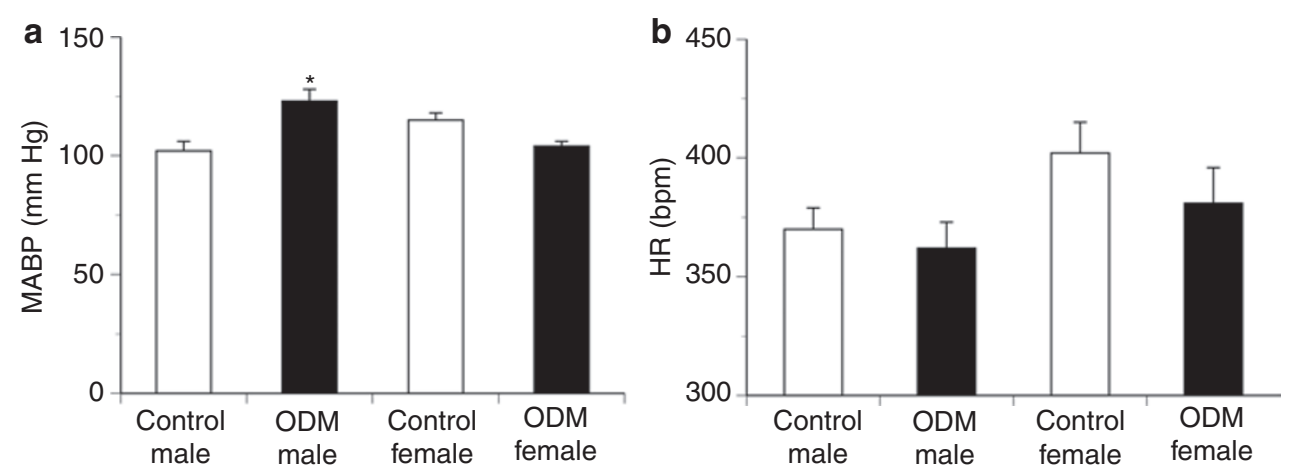

Figure 1. Resting (a) mean arterial blood pressure (MABP) and (b) heart rate (HR) in control and offspring of diabetic mothers (ODM) male and female adult rats. Data are reported as mean \pm SE. bpm, beats per minute. ${ }^{*} P<0.01$ as compared with control male. 

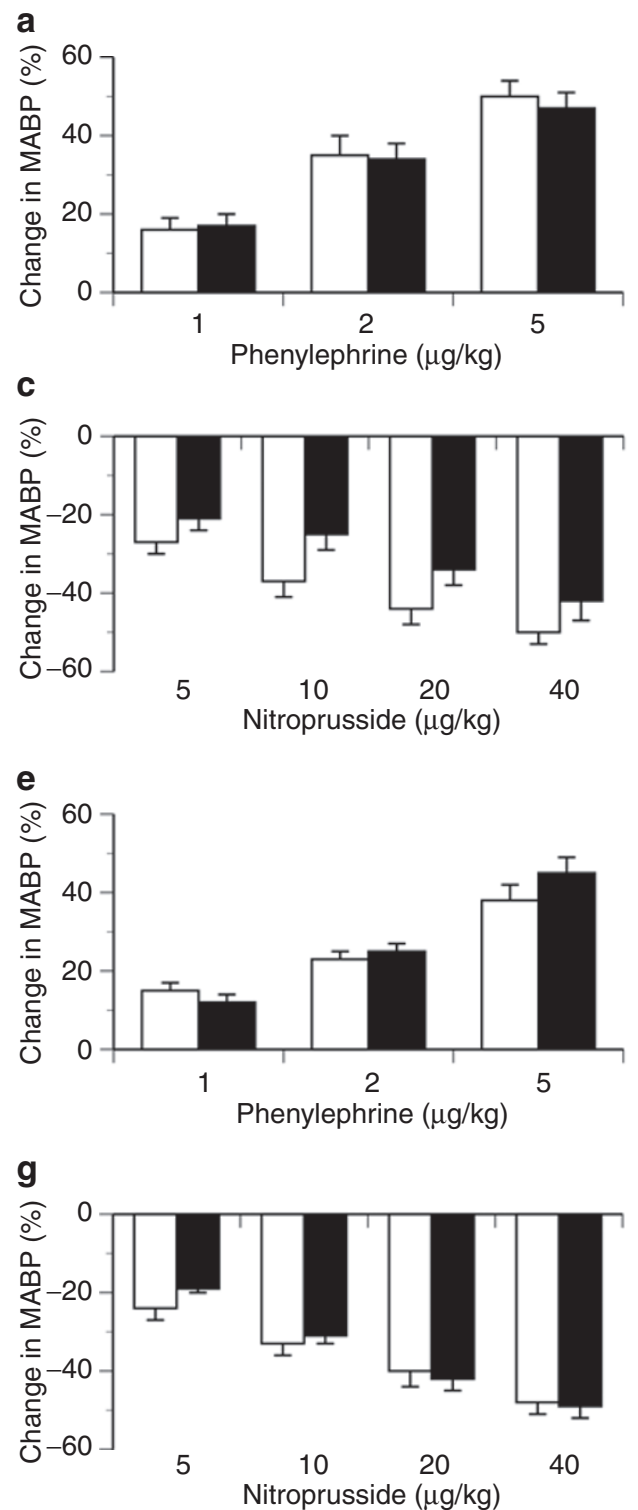

b

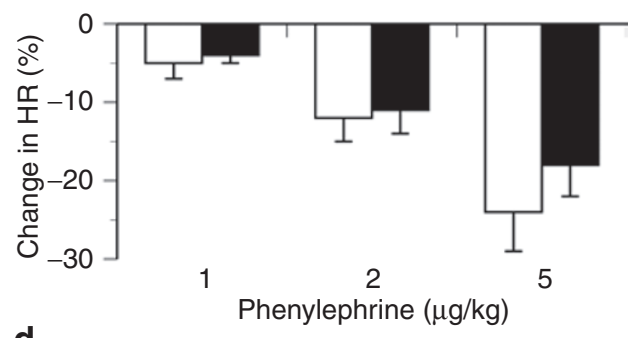

d

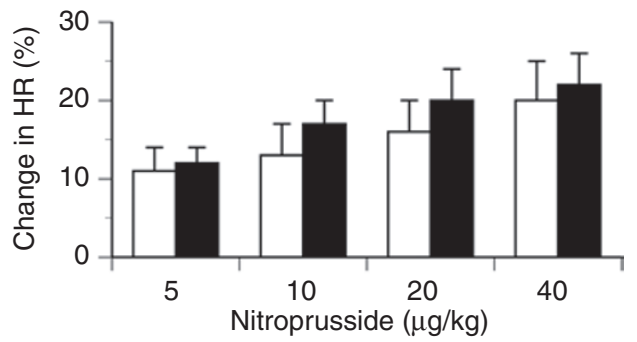

f
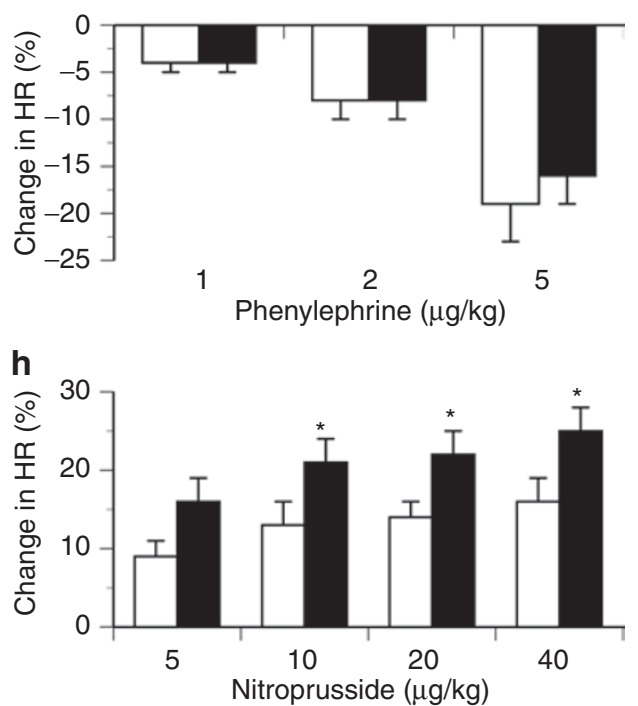

Figure 2. Changes in systemic hemodynamics in response to increasing doses of phenylephrine and nitroprusside in male (top two rows) and female (bottom two rows) control (open bars) and offspring of diabetic mothers (filled bars) adult rats. (a) Mean arterial blood pressure (MABP) responses to phenylephrine in males. (b) Heart rate (HR) responses to phenylephrine in males. (c) MABP responses to nitroprusside in males. (d) HR responses to nitroprusside in males. (e) MABP responses to phenylephrine in females. (f) HR responses to phenylephrine in females. (g) MABP responses to nitroprusside in females. (h) HR responses to nitroprusside in females. Data are reported as mean $\pm \mathrm{SE}$. ${ }^{*} P<0.05$ as compared with control females for similar dose.

superoxide production was 1.5-2-fold higher in aorta from male ODM as compared with control, whereas in females, NADPHstimulated superoxide production was similar between groups (Figure 7).

\section{DISCUSSION}

It is increasingly being recognized that exposure to hyperglycemia in utero results in long-term consequences, including the development of vascular dysfunction and hypertension $(9,13,14)$. The majority of animal studies evaluating the effects of hyperglycemia during pregnancy have studied offspring of mothers made diabetic before or relatively early in gestation and were reared by these same mothers (i.e., not cross-fostered). As such, a critical window of exposure to maternal diabetes resulting in cardiovascular morbidity in the offspring has not been established and could potentially occur during the late prenatal or postnatal period. We evaluated hemodynamics and vascular responsiveness in offspring exposed to maternal hyperglycemia only in the last third of pregnancy, similar to that occurring with gestational diabetes. Furthermore, all rat pups were cross-fostered to nondiabetic mothers, effectively removing the postnatal period as a contributor to the offspring phenotype. The results of these studies demonstrate that exposure to hyperglycemia late in gestation results in hypertension and increased NADPH-stimulated superoxide production in male but not female offspring at 6-12 mo of age. However, ODM of both sexes displayed abnormalities in aortic vascular reactivity related to altered $\mathrm{NO}$ and reactive oxygen species (ROS) signaling/bioavailability. 
a

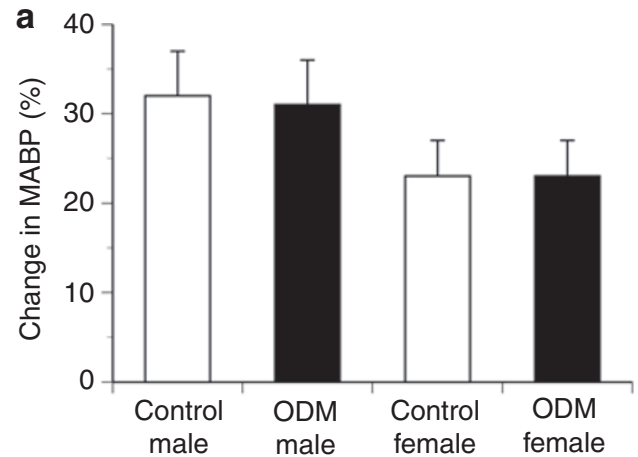

c

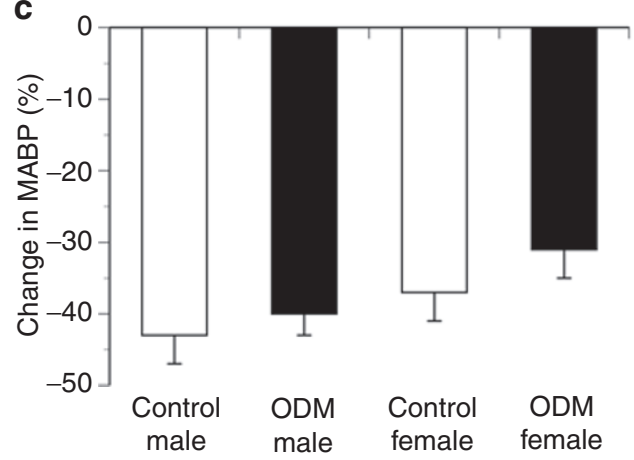

b

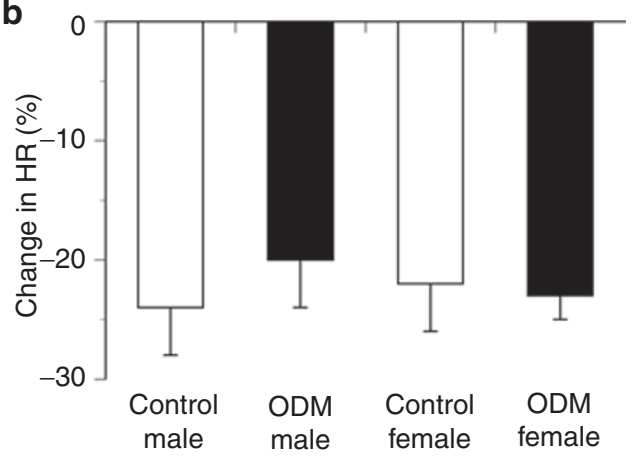

d

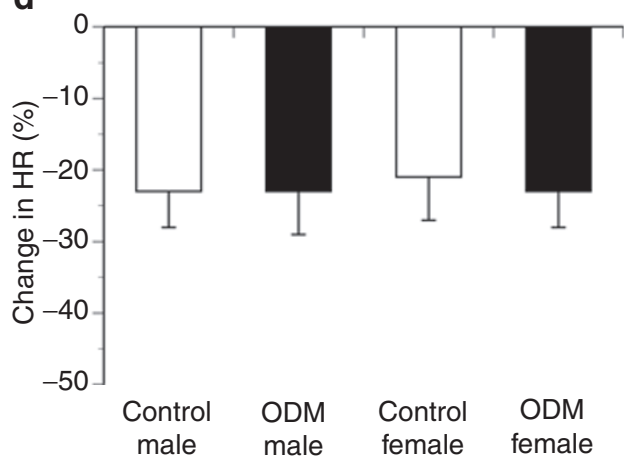

Figure 3. Changes in systemic hemodynamics (expressed as percentage change from baseline) to $\mathrm{N}^{\mathrm{G}}$-nitro-L-arginine methyl ester (L-NAME) (top panels) and chlorisondamine (bottom panels) in control and offspring of diabetic mothers male and female animals. (a) Change in mean arterial blood pressure (MABP) in response to L-NAME. (b) Change in heart rate (HR) in response to L-NAME. (c) Change in MABP in response to chlorisondamine. (d) Change in HR in response to chlorisondamine. Data are reported as mean $\pm \mathrm{SE}$.

In a previous study using this same animal model, we showed that female but not male ODM exhibited vascular and endothelial dysfunction, as evidenced by increased aortic contractile responses to endothelin-1 and noradrenaline, and decreased vasorelaxation in response to the endothelial dependent vasodilator, acetylcholine (17). Therefore, we were surprised to observe that males, but not females, were hypertensive at 6-12 mo of age. Of note, sex-specific hypertension has been shown in a variety of developmental programming models. Both moderate maternal protein restriction and uteroplacental insufficiency result in hypertension in male but not female offspring (21-23). The etiology of sex differences in developing hypertension is unclear, although potential mechanisms, including differences in the amounts of sex hormones or fundamental differences at the level of gene expression, have recently been reviewed (24).

We used in vivo assessment of systemic hemodynamics and vascular function to identify components of neurohumoral regulation of cardiovascular function that may contribute to hypertension in male ODM. Baroreflex dysfunction has been observed in hypertension both as a primary cause and a secondary effect (25). In a model of maternal diabetes in which pregnant dams were made diabetic by STZ on the 7th day of pregnancy, Wichi et al. (14) found increased blood pressure responses to phenylephrine and impaired baroreflex mediated tachycardia elicited by decreasing MABP with nitroprusside in male offspring. In contrast, we found no differences in dose response changes in MABP and HR to phenylephrine and nitroprusside or the slope of the baroreflex response to either increasing or decreasing blood pressure between male ODM and control animals, suggesting the baroreflex function remains intact. Reasons for the discrepancy in findings between our study and those of Wichi et al. (14) may relate to differences in age of offspring, duration of maternal diabetes, or timing of the studies related to surgical catheter placement. However in females, the HR baroreflex response to unloading baroreceptors, elicited by decreasing blood pressure with nitroprusside, tended to be greater in ODM as compared with controls. Specifically, nitroprusside injection elicited significantly greater increases in HR $(10,20$, and $40 \mu \mathrm{g} / \mathrm{kg})$ in ODM as compared with control animals, although decreases in blood pressure were similar. HR baroreflex sensitivity also tended to be different $(P=0.06)$, although failure to reach significance related to the slight but not significantly different decreases in blood pressure between groups. The finding of increased sensitivity of the baroreflex is opposite that expected in a prehypertensive state, where impairment (decreased sensitivity) of the baroreflex is likely to be observed. Without further study, it is not possible to say whether this effect was mediated at the level of the baroreceptor or was related to central processing of the afferent signal or to end-organ response differences.

We previously found evidence for aortic endothelial dysfunction in female ODM, evidenced by decreased vasorelaxation to acetylcholine, and attenuation of the increased vasoconstrictive responses to endothelin-1 and noradrenaline in ODM as compared with controls by removal of endothelium or following 

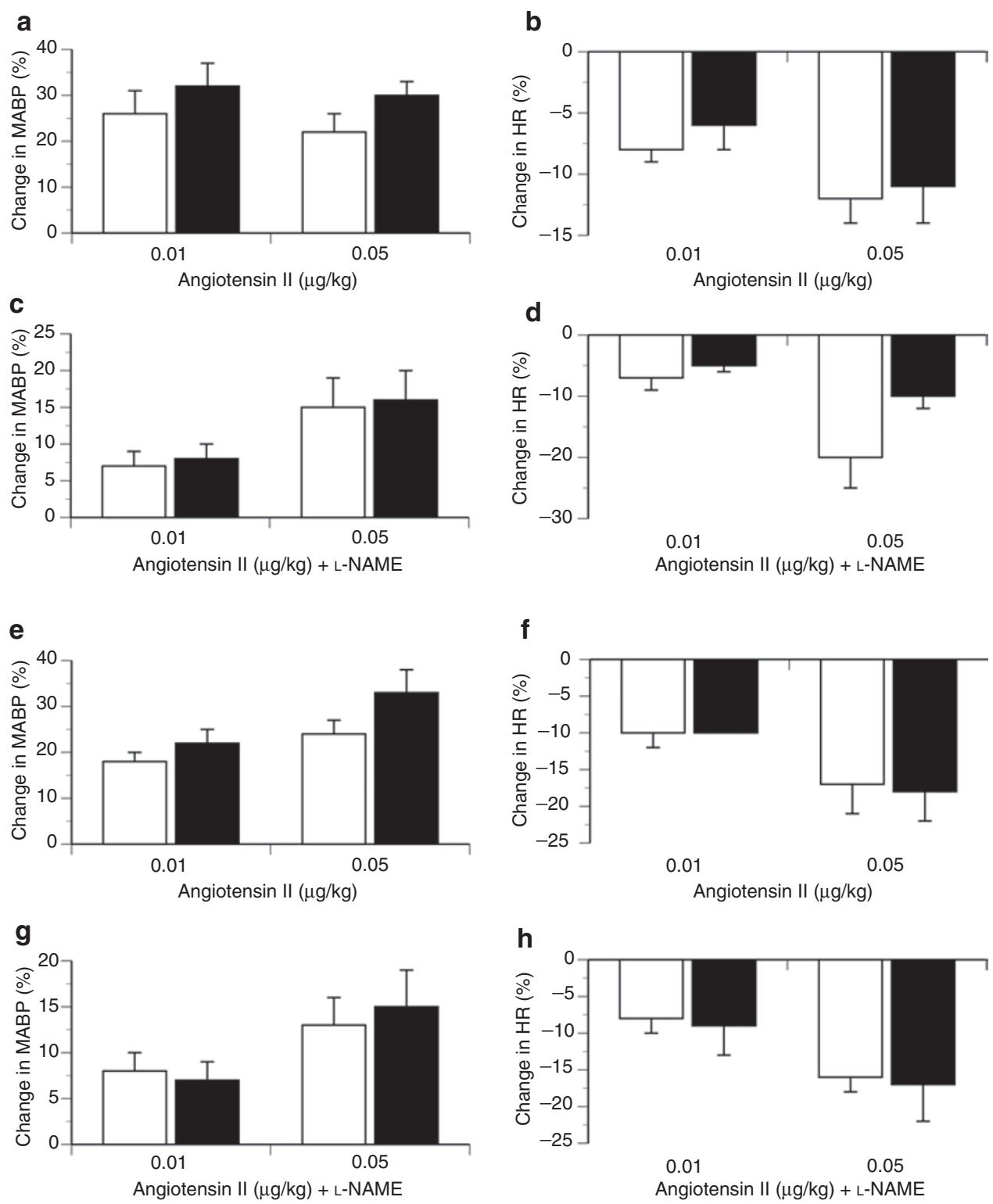

Figure 4. Changes in systemic hemodynamics (expressed as percentage change from baseline) to increasing doses of angiotensin II before and after administration of $\mathrm{N}^{\mathrm{G}}$-nitro-L-arginine methyl ester (L-NAME) (10 mg/kg i.v.) in male (top two rows) and female (bottom two rows) control (open bars) and offspring of diabetic mothers (filled bars) adult rats. (a) Mean arterial blood pressure (MABP) responses to angiotensin II in males. (b) Heart rate (HR) responses to angiotensin II in males. (c) MABP responses to angiotensin II + L-NAME in males. (d) HR responses to angiotensin II + L-NAME in males. (e) MABP responses to angiotensin II in females. (f) HR responses to angiotensin II in females. (g) MABP responses to angiotensin II + L-NAME in females. (h) HR responses to angiotensin II + L-NAME in females. Data are reported as mean \pm SE.

L-NNA exposure (17). Cavanal et al. (26) also found basal aortic NO production was decreased in ODM as compared with controls. In contrast to these findings in isolated vessels, we found no differences in the pressor response following L-NAME treatment, suggesting endogenous NO bioavailability and function was similar between groups. To further interrogate the NO buffering system that may mask a predisposition to hypertension, we examined pressor responses to bolus doses of angiotensin II before and after L-NAME. No significant differences in the blood pressure responses between treatment groups in either sex were identified, again suggesting similarly intact NO signaling pathways in controls and ODM. The findings demonstrate that abnormalities in endothelial function and NO bioavailability in the aorta may not, in fact, be present throughout the vascular bed, particularly in resistance vessels that contribute to maintenance of blood pressure.

Sympathetic function is essential to blood pressure regulation, and overactivity of sympathetic nerves may have an important role in the development of hypertension and related cardiovascular disorders (25). In many experimental models of hypertensive rats, there appears to be an important contribution from the sympathetic nervous system. A number of models of developmental programming of hypertension also suggest an important role of sympathetic function contributing to 

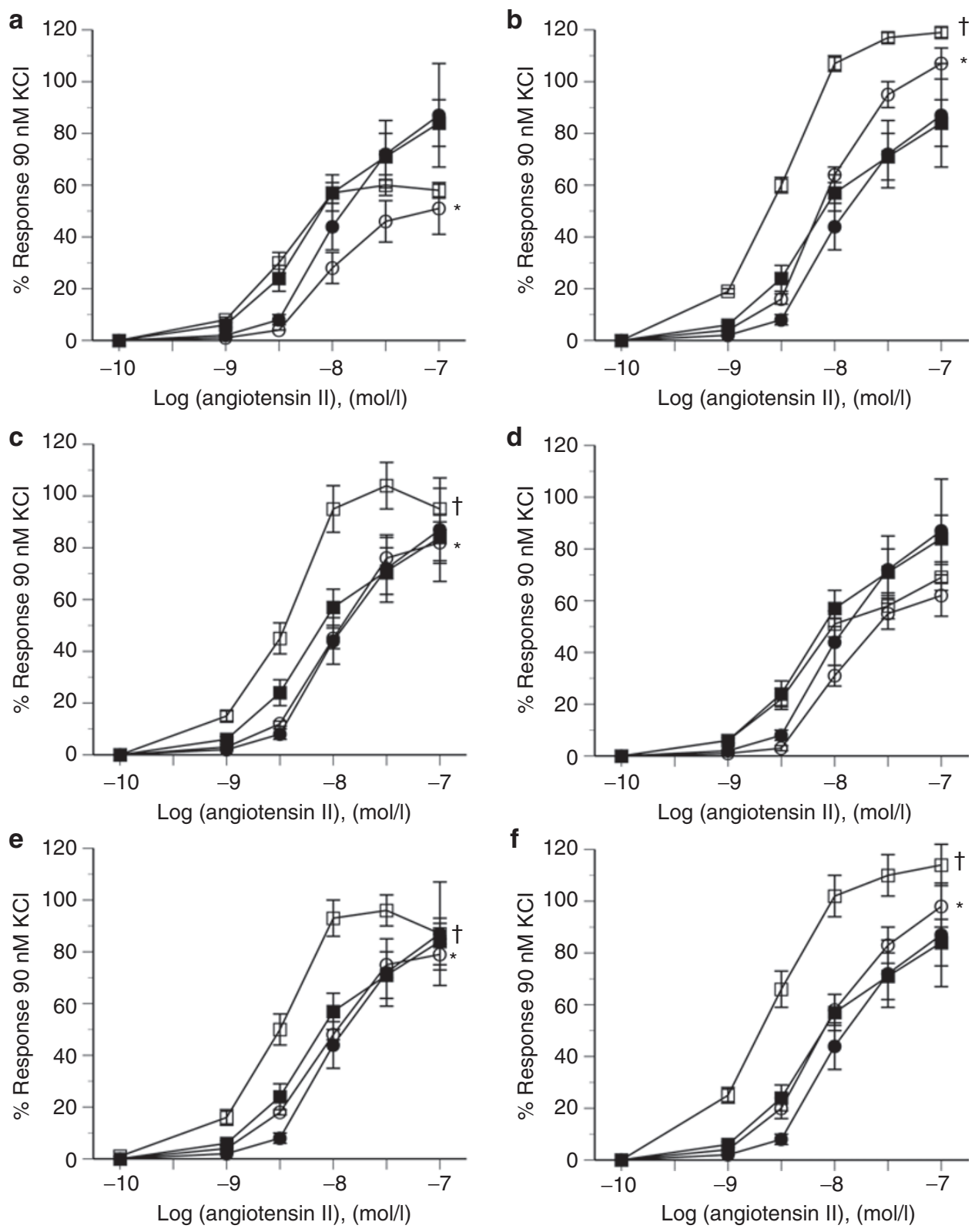

Figure 5. Male aortic responsiveness to angiotensin II in the absence and presence of (a) PD 123319, (b) NG'-nitro-L-arginine (L-NNA), (c) diethyldithiocarbamate (DETC), (d) Tempol, (e) L-NNA + DETC, and (f) L-NNA + Tempol. In each panel: angiotensin alone in controls (filled squares) and offspring of diabetic mothers (ODM) (filled circles); angiotensin in presence of specific agent in controls (open squares) and ODM (open circles). Data are reported as mean \pm SE. ${ }^{*} P<0.05$ between control and ODM in the presence of specific agent in each panel (treatment effect by ANOVA); $+P<0.05$ between control in the absence and presence of specific agent (treatment effect by ANOVA).

the phenotype (27-29). To determine the contribution of the sympathetic drive to the maintenance of blood pressure, we administered the ganglionic blocking agent chlorisondamine. Impairment of the sympathetic drive by blocking ganglionic transmission results in a rapid blood pressure fall, which is related to the degree of sympathetic activity. We hypothesized that male ODM would exhibit a larger decrease in blood pressure in response to chlorisondamine as compared with controls, suggesting increased sympathetic vascular tone in this group. However, the blood pressure decreases associated with chlorisondamine, expressed both as percentage change or absolute change in $\mathrm{mm} \mathrm{Hg}$ (data not shown), were not different in ODM as compared with control male or female animals. We recognize these findings do not rule out a significant contribution of the sympathetic nervous system in contributing to hypertension in ODM. For example, increased renal sympathetic nerve activity may alter renal excretory function and thus contribute to altered renal sodium handling and ultimately hypertension. Along these lines, it has previously been shown that offspring of rats with STZ-induced diabetes beginning on day 0 of gestation have reduced nephron number, salt-sensitive hypertension, and altered renal sodium handling (30). Further studies are needed to see if a similar phenotype is present in our model of maternal diabetes. 

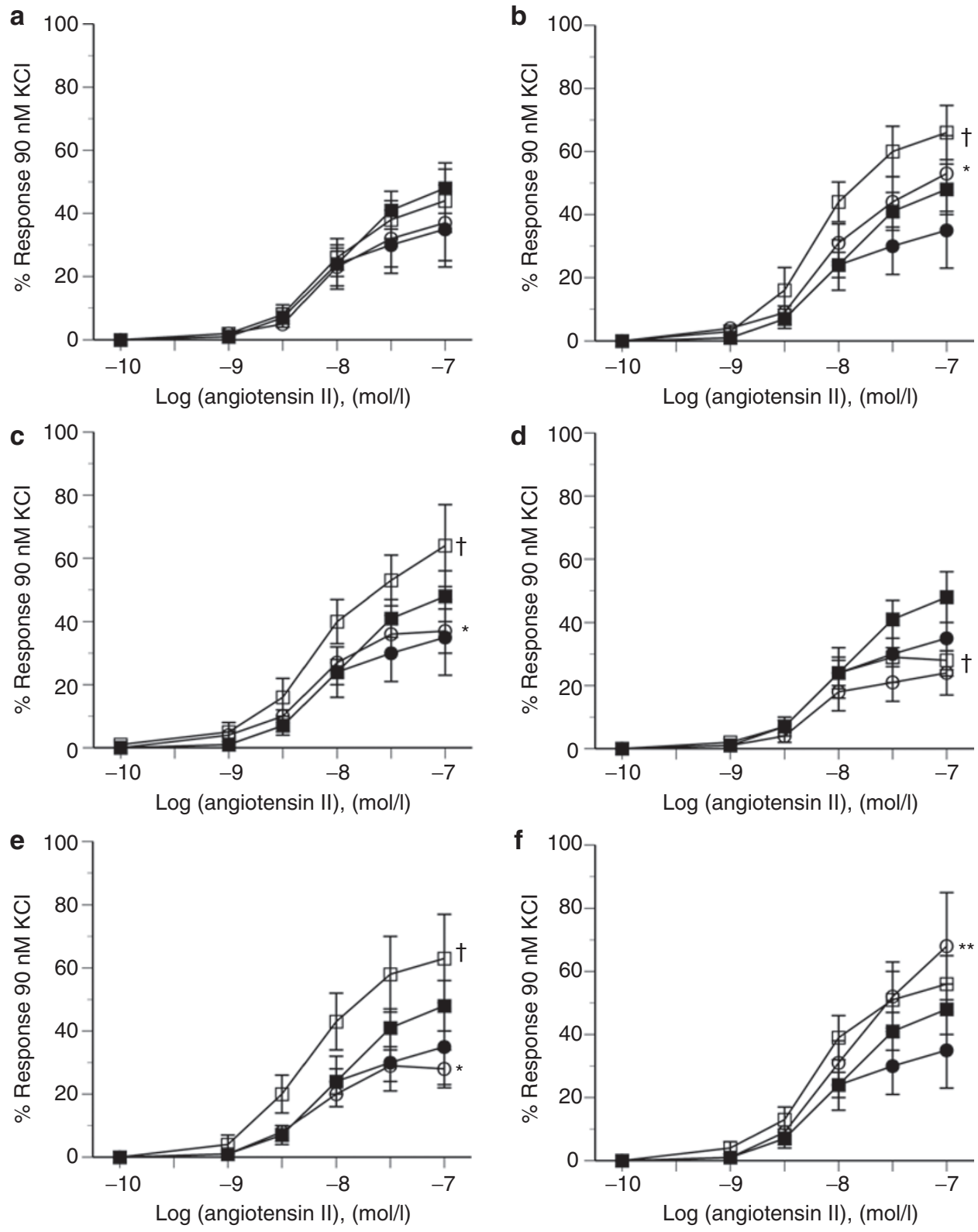

Figure 6. Female aortic responsiveness to angiotensin II in the absence and presence of (a) PD 123319, (b) NG-nitro-L-arginine (L-NNA), (c) diethyldithiocarbamate (DETC), (d) Tempol, (e) L-NNA + DETC, and (f) L-NNA + Tempol. In each panel: angiotensin alone in controls (filled squares) and offspring of diabetic mothers (ODM) (filled circles); angiotensin in the presence of specific agent in controls (open squares) and ODM (open circles). Data are reported as mean \pm SE. ${ }^{*} P<0.05$ between control and ODM in the presence of specific agent in each panel (treatment effect by ANOVA); $+P<0.05$ between control in the absence and presence of specific agent (treatment effect by ANOVA); ${ }^{* *} P<0.05$ between ODM in the absence and presence of specific agent (treatment effect by ANOVA).

Using ex vivo wire myography, we found inhibition of NO synthase by L-NNA resulted in enhanced angiotensin II-mediated vasoconstriction in control but not ODM animals, findings consistent with those we previously reported in this model and suggesting impaired NO bioavailability in ODM. Whether this is related to decreased levels of the substrate $\mathrm{L}$-arginine, $\mathrm{NO}$ synthase, uncoupling of NO synthase, or scavenging by increased levels of superoxide remains to be determined (26). A number of studies have demonstrated that a suboptimal uterine environment, including maternal obesity, diabetes, and preeclampsia, alters oxidative pathways in the placenta and offspring (31-33).
Evidence of oxidative stress and disruption of oxidative signaling remains well into the postnatal period and adulthood. Using lucigenin-enhanced chemiluminescence, we found that aortas from male but not female ODM generated 1.5-2-fold higher amounts of superoxide in response to NADPH than did controls. In addition to smooth muscle contraction and vascular tone, ROS mediate a number of vascular physiologic functions, including transcription factor activation, gene expression, cell growth, and apoptosis. Thus, conclusions about the effects of chronic exposure to elevated superoxide levels are difficult, although ROS-mediated cellular injury is thought to contribute 

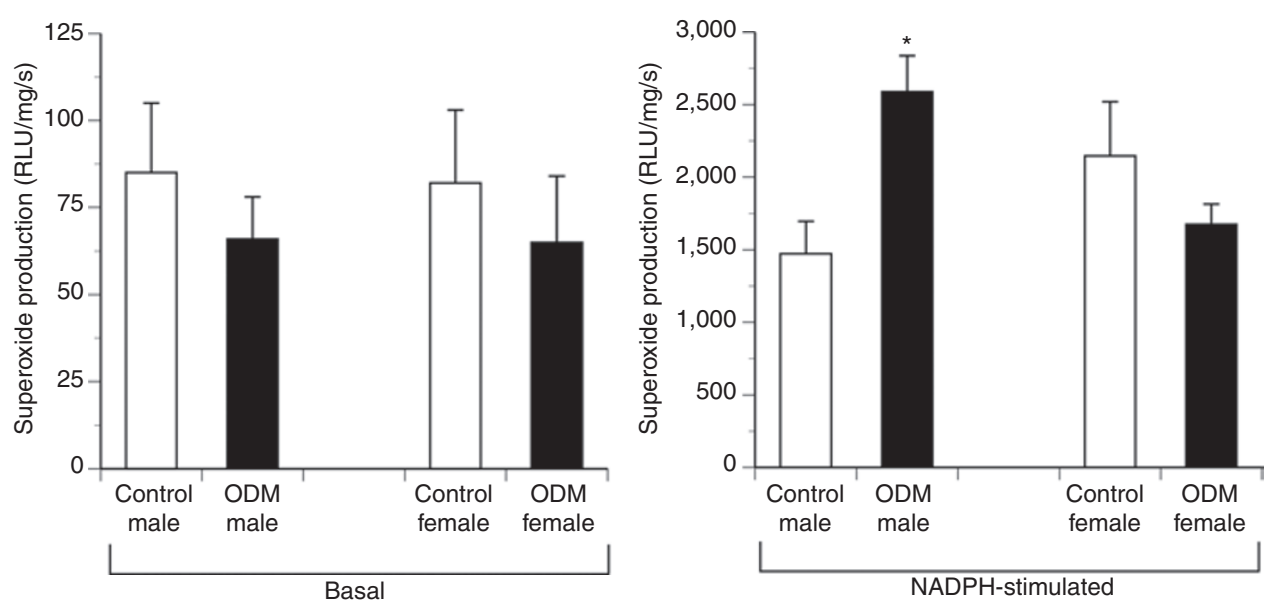

Figure 7. Basal and reduced nicotinamide adenine dinucleotide phosphate oxidase-stimulated superoxide production determined by lucigeninenhanced chemiluminescence of abdominal aorta from control animals and offspring of diabetic mothers. Values reported in relative light units (RLU)/mg dry weight of tissue/s. Data are reported as mean \pm SE. ${ }^{*} P<0.05$ as compared with control male.

to cardiovascular disease. The acute effects of altered ROS species are more easily examined, although they remain complex. In endothelial-denuded rat aortic rings, Shen et al. demonstrated that different ROS species induced contraction via separate mechanisms of action (34). Using the superoxide generator pyrogallol, Demirci et al. demonstrated that the effects on endothelium-dependent vasodilation were dose dependent, improving Ach-mediated relaxation at low concentration but attenuating responses at higher concentrations (35). A similar dose-dependent effect was seen when phenylephrine-induced contractions were studied, with low doses of pyrogallol diminishing contraction and high doses enhancing contractility. In our study, we found Tempol had no significant effect on angiotensin II-mediated contractions. On the other hand, DETC significantly enhanced contractions in control but not ODM animals. We speculate that in control animals, with intact and functional endothelium, DETC resulted in a small increase in vascular superoxide levels that enhanced contraction. In contrast, in ODM, in which endothelial dysfunction is already present and superoxide levels are elevated, DETC has little effect on contractile responses. Because studies were not performed in endothelial-denuded vessels, we cannot comment on potential direct effects of superoxide or other ROS on smooth muscle cells in this model.

There are several limitations of the study. First, offspring were studied over a range of ages $(6-12 \mathrm{mo})$, and it is possible that agerelated effects exist but were not identified. Certainly, some of the difficulties in reconciling our findings with those of previous studies may relate to the large age range of the rats used in our studies. An age-related effect of increased blood pressure (seen in male ODM) on composition and function of the arterial wall may also be more pronounced in older vs. younger adult rats and was not accounted for in the experimental design (36). Second, vascular reactivity and superoxide production studies were performed in the aorta, a conduit vessel, and not in resistance vessels, where alterations in vascular function likely have a more physiologic contribution to the maintenance of blood pressure.
Finally, because we used STZ to induce maternal diabetes, our model is one of late-gestation insulinopenia and hyperglycemia and not gestational diabetes, a condition of insulin resistance.

In summary, we showed that exposure to maternal diabetes during the last third of gestation results in hypertension in adult male but not female offspring. Results of the in vivo studies, examining baroreflex function, the NO contribution to blood pressure, and vascular sympathetic tone, failed to identify abnormalities in these systems that may directly contribute to hypertension. However, oxidative stress may contribute to the underlying phenotypes in ODM as the maternal diabetic in utero environment results in increased vascular superoxide production in male offspring and endothelial dysfunction and impaired NO-ROS signaling in offspring of both sexes.

\section{METHODS}

\section{Animal Preparation}

All procedures were performed within the regulations of the Animal Welfare Act and the National Institutes of Health Guide for the Care and Use of Laboratory Animals, and were approved by the Institutional Animal Care and Use Committee of the University of Iowa. Pregnant Sprague-Dawley rats (Charles River Laboratories, Wilmington, MA) received an i.p. injection of either STZ (Sigma-Aldrich, St. Louis, MO) $(50 \mathrm{mg} / \mathrm{kg}$ of body weight in $10 \mathrm{mmol} / \mathrm{l}$ citrate buffer $(\mathrm{pH} \mathrm{3.5})$ or an equivalent volume of citrate buffer alone on gestational day 13. STZinjected rats had blood glucose measured twice daily via tail-nicking, using the LifeScan OneTouch Ultra Blood Glucose Monitoring System (LifeScan, Milpitas, CA). Hyperglycemia was partially controlled and ketosis was avoided by subcutaneous injection of insulin (Humulin; Eli Lilly, Indianapolis, IN) in the morning and insulin-glargine (Lantus; Sanofi-Aventis, Bridgewater, NJ) each evening to maintain blood glucose between 100 and $400 \mathrm{mg} / \mathrm{dl}$. Control rats were injected with saline twice daily. Dams were allowed to deliver spontaneously. All pups were cross-fostered with nondiabetic postpartum dams. Litters were culled to 10 pups, selecting to preserve the largest pups. Pups of both sexes were weaned onto standard rat chow on day 21 . We have previously described this methodological approach and details regarding maternal glycemic control and the metabolic status of the offspring (17).

\section{In Vivo Experimental Protocol}

At 6-12 mo of age, offspring exposed to late-gestation hyperglycemia and offspring of control mothers of both sexes were studied $(n=6-8$ 
for each sex and each condition). This was a wide range in ages of animals studied, however, we think this is a valid grouping based on (i) the fact that the distribution of ages of the animals was similar in all groups and (ii) several groups have shown that systolic blood pressure, $\mathrm{HR}$, nitroprusside and acetylcholine-induced relaxation of aortic rings are unchanged in young adult and old adult WKY rats (37-39). Anesthesia was induced and maintained with inhalational isoflurane mixed with oxygen. The left femoral triangle was opened, a polyethylene catheter (PE 50, Intramedic, Franklin Lakes, NJ) was inserted and secured into the femoral artery, while a PE 10 catheter was inserted and secured into the femoral vein. Catheters were tunneled subcutaneously to a mid-scapular exit and connected to a Covance Infusion Harness (Instech Laboratories, Plymouth Meeting, PA), which allows the rat freedom of movement. Postoperative analgesia included bupivacaine (International Laboratory, San Bruno, CA) placed topically along suture lines before closing the skin. Normal saline with $25 \mathrm{U} / \mathrm{ml}$ heparin was continuously infused at $5 \mu \mathrm{l} / \mathrm{min}$ in the arterial catheter to maintain patency whereas the venous catheter was filled with $0.05 \mathrm{ml}$ of $0.9 \%$ $\mathrm{NaCl}$ with $500 \mathrm{U} / \mathrm{ml}$ heparin. After surgery, animals were allowed free access to food and water. Three days were allowed for recovery from surgery before experiments were performed.

Arterial blood pressure was recorded using Statham P23 Db pressure transducers (Spectramed, Critical Care Division, Oxnard, CA), MacLab hardware and software (ADInstruments, Colorado Springs, CO), and a G4 Powerbook (Apple Computers, Cupertino, CA). HR was monitored with a cardiotachometer triggered from the arterial pressure pulse wave. On the first study day, baseline blood pressure and HR were determined. We then examined the function of the arterial baroreflex by measurement of dose response changes in MABP and HR to phenylephrine $(1,2$, and $5 \mu \mathrm{g} / \mathrm{kg}$, i.v.) and sodium nitroprusside $(5,10,20$, and $40 \mu \mathrm{g} / \mathrm{kg}$, i.v.). A $20-30 \mathrm{~min}$ recovery period was allowed for MABP and HR to return to baseline before administration of each subsequent dose. All agents were given intravenously as a bolus injection, with the volume adjusted to $0.1 \mathrm{ml}$.

On the following day, dose response blood pressure and HR changes in response to angiotensin II $(0.05$ and $0.1 \mu \mathrm{g} / \mathrm{kg}$, i.v. $)$, were examined to assess functional response to angiotensin receptor stimulation. After a 1 -h recovery period, baseline blood pressure and HR were again determined followed by administration of the NO synthase inhibitor L-NAME ( $10 \mathrm{mg} / \mathrm{kg}$, i.v., Sigma-Aldrich). This approach allows assessment of the contribution of NO to maintenance of resting blood pressure. A dose response to angiotensin II $(0.01$ and $0.05 \mu \mathrm{g} / \mathrm{kg}$, i.v. $)$ was again performed, allowing assessment of the contribution of NO to buffering the pressor response resulting from angiotensin II. On the final day of study, rats received the ganglionic blocker chlorisondamine $(5 \mathrm{mg} / \mathrm{kg}$, i.v.). This approach allows us to assess the contribution of vascular sympathetic tone to the maintenance of baseline blood pressure.

All cardiovascular measurements were performed in conscious, freely moving rats. Baseline blood pressure and HR were reported as an average over time (at least $30 \mathrm{~min}$ ). All drugs were given i.v. as a bolus injection, with the volume of agent adjusted to $0.1 \mathrm{ml}$. The doses used for the various pharmacologic agents administered in vivo were chosen based on review of the literature and preliminary studies examining the range of responses to the agents (40-42). Hemodynamic responses to increasing doses of phenylephrine, sodium nitroprusside, and angiotensin II are reported as a maximal percentile change from resting blood pressure and HR immediately before giving the drugs. Subsequent injections were given only after hemodynamics had returned to basal levels.

\section{Ex Vivo Vascular Reactivity}

In a separate group of animals $(n=6-7$ for each sex and each condition), aortic vascular reactivity was assessed using methods previously described (17). Briefly, offspring were killed and descending thoracic aorta segments harvested, cleansed of adherent connective tissue and sectioned into 2-mm-long rings. Aortic rings were mounted in individual 18-ml isolated organ chambers (Radnoti Glass Technology, Monrovia, $\mathrm{CA}$ ) and connected to an isometric force transducer. Contractile responses were recorded with a MacLab 8E (ADInstruments, Colorado Springs, CO) and stored on a Power Macintosh 8600 computer. Passive stretch was set at $90 \%$ of the tension required to obtain peak responses to potassium chloride ( $2.5 \mathrm{~g}$, determined in preliminary studies) and the rings were allowed to equilibrate in bicarbonate-buffered physiological salt solution at $37^{\circ} \mathrm{C}$ for $60 \mathrm{~min}$ before the start of experimentation. The composition of the physiological salt solution was as follows (in mmom/l): $130 \mathrm{NaCl}, 4.7 \mathrm{KCl}, 1.18 \mathrm{KH}_{2} \mathrm{PO}_{4}, 1.17 \mathrm{MgSO}_{4}, 7 \mathrm{H} \mathrm{O}_{2}$, $14.9 \mathrm{NaHCO}_{3}, 1.6 \mathrm{CaCl}_{2} \cdot \mathrm{H}_{2} 0,5.5$ dextrose and $0.03 \mathrm{CaNa}_{2}-\mathrm{EDTA}(\mathrm{pH}$ 7.30). Physiological salt solution was aerated with a mixture of $95 \%$ $\mathrm{O}_{2}-5 \% \mathrm{CO}_{2}$.

To evaluate the separate and combined roles of angiotensin type 1 and type 2 receptors, superoxide production, and nitric oxide production on the vasoconstrictive effects of angiotensin II, aortic vessel segments were incubated, respectively, with the angiotensin II type 1 receptor antagonist losartan $\left(10^{-6} \mathrm{~mol} / \mathrm{l}\right)$, the angiotensin II type 2 receptor antagonist $\mathrm{PD}-123319\left(10^{-7} \mathrm{~mol} / \mathrm{l}\right)$, the superoxide dismutase mimetic Tempol $(1 \mathrm{mmol} / \mathrm{l})$, the $\mathrm{Cu} / \mathrm{Zn}$ superoxide dismutase inhibitor DETC $(10 \mathrm{mmol} / \mathrm{l})$, the NO synthase inhibitor L-NNA $\left(10^{-4} \mathrm{~mol} / \mathrm{l}\right)$, Tempol + L-NNA, and DETC + L-NNA. Separate baths were used to assess the cumulative concentration responses to angiotensin II $\left(10^{-11}\right.$ to $10^{-7} \mathrm{~mol} / \mathrm{l}$ ) in the absence and presence of the agents described above. All physiological salt solution reagents and vasoactive compounds were acquired from Sigma-Aldrich. Doses of pharmacologic agents were determined based on our previous experience with these agents and literature review $(43,44)$. Angiotensin II was used as the constriction agent as our previous studies demonstrated no differences in constriction between aortas obtained from control and ODM animals. This approach allowed us to be confident that any measured differences in the constrictive response to angiotensin II was related to the presence of the additional pharmacologic agent(s) added to the bath.

\section{Chemiluminescence}

To assess the effects of in utero exposure to hyperglycemia on vascular ROS, basal superoxide anion production was measured by lucigenin $(25 \mu \mathrm{mol} / \mathrm{l})$-enhanced chemiluminescence after $5 \mathrm{~min}$ of dark adaptation as previously described (luminometer model FB12; Zylux, Huntsville, AL) (45). Proximal abdominal aorta excised from animals undergoing ex vivo vascular reactivity studies and sectioned into $3 \mathrm{~mm}$ rings was used. NADPH oxidase-dependent superoxide production was measured as the diphenylene-iodonium $\left(10^{-4} \mathrm{~mol} / \mathrm{l}\right)$-inhibitable chemiluminescence measured after the addition of the enzyme substrate NADPH $\left(10^{-4}\right.$ $\mathrm{mol} / \mathrm{l})$. Superoxide anion production, as determined by chemiluminescent relative light units, was normalized to weight of the aortic segment.

\section{Data Analysis}

For each animal, baroreflex sensitivity was determined by calculating the slope of the linear regression relating the percentage change in MABP to percentage change in HR. Animals of each sex were analyzed separately. Comparisons between groups were performed by Student's unpaired, two-tailed $t$-test or ANOVA, with factor for treatment group and drug concentration. If ANOVA identified significant differences, defined as $P<0.05$, pairwise comparisons were made using the Tukey test, with $P<0.05$ considered significant. Variances were compared using the variance ration test (F-test). All results are expressed as means \pm SE.

\section{STATEMENT OF FINANCIAL SUPPORT}

This work was supported by a grant from Ikaria, Inc. (to R.K.), National Institutes of Health (NIH) grant DK077599 (to T.D.S.,) and NIH grant R01 DK081548 (to A.W.N.).

Disclosure: The authors declared no conflict of interest.

\section{REFERENCES}

1. Dabelea D, Snell-Bergeon JK, Hartsfield CL, Bischoff KJ, Hamman RF, McDuffie RS; Kaiser Permanente of Colorado GDM Screening Program. Increasing prevalence of gestational diabetes mellitus (GDM) over time and by birth cohort: Kaiser Permanente of Colorado GDM Screening Program. Diabetes Care 2005;28:579-84.

2. Shaw J. Epidemiology of childhood type 2 diabetes and obesity. Pediatr Diabetes 2007;8:Suppl 9:7-15.

3. Nold JL, Georgieff MK. Infants of diabetic mothers. Pediatr Clin North Am 2004;51:619-37, viii. 
4. Dabelea D. The predisposition to obesity and diabetes in offspring of diabetic mothers. Diabetes Care 2007;30:Suppl 2:S169-74.

5. Lee H, Jang HC, Park HK, Cho NH. Early manifestation of cardiovascular disease risk factors in offspring of mothers with previous history of gestational diabetes mellitus. Diabetes Res Clin Pract 2007;78: 238-45.

6. Manderson JG, Mullan B, Patterson CC, Hadden DR, Traub AI, McCance DR. Cardiovascular and metabolic abnormalities in the offspring of diabetic pregnancy. Diabetologia 2002;45:991-6.

7. Metzger BE. Long-term outcomes in mothers diagnosed with gestational diabetes mellitus and their offspring. Clin Obstet Gynecol 2007;50: 972-9.

8. Vohr BR, Boney CM. Gestational diabetes: the forerunner for the development of maternal and childhood obesity and metabolic syndrome? J Matern Fetal Neonatal Med 2008;21:149-57.

9. Bunt JC, Tataranni PA, Salbe AD. Intrauterine exposure to diabetes is a determinant of hemoglobin $A(1) c$ and systolic blood pressure in pima Indian children. J Clin Endocrinol Metab 2005;90:3225-9.

10. Cho NH, Silverman BL, Rizzo TA, Metzger BE. Correlations between the intrauterine metabolic environment and blood pressure in adolescent offspring of diabetic mothers. J Pediatr 2000;136:587-92.

11. Mansford KR, Opie L. Comparison of metabolic abnormalities in diabetes mellitus induced by streptozotocin or by alloxan. Lancet 1968;1:670-1.

12. Holemans K, Gerber RT, Meurrens K, De Clerck F, Poston L, Van Assche FA. Streptozotocin diabetes in the pregnant rat induces cardiovascular dysfunction in adult offspring. Diabetologia 1999;42:81-9.

13. Rocha SO, Gomes GN, Forti AL, et al. Long-term effects of maternal diabetes on vascular reactivity and renal function in rat male offspring. Pediatr Res 2005;58:1274-9.

14. Wichi RB, Souza SB, Casarini DE, Morris M, Barreto-Chaves ML, Irigoyen MC. Increased blood pressure in the offspring of diabetic mothers. Am J Physiol Regul Integr Comp Physiol 2005;288:R1129-33.

15. Duong Van Huyen JP, Vessières E, Perret C, et al. In utero exposure to maternal diabetes impairs vascular expression of prostacyclin receptor in rat offspring. Diabetes 2010;59:2597-602.

16. Gomes GN, Gil FZ. Prenatally programmed hypertension: role of maternal diabetes. Braz J Med Biol Res 2011;44:899-904.

17. Segar EM, Norris AW, Yao JR, et al. Programming of growth, insulin resistance and vascular dysfunction in offspring of late gestation diabetic rats. Clin Sci 2009;117:129-38.

18. Gordon FJ, Matsuguchi H, Mark AL. Abnormal baroreflex control of heart rate in prehypertensive and hypertensive Dahl genetically salt-sensitive rats. Hypertension 1981;3(3 Pt 2):I135-41.

19. Biri A, Onan A, Devrim E, Babacan F, Kavutcu M, Durak I. Oxidant status in maternal and cord plasma and placental tissue in gestational diabetes. Placenta 2006;27:327-32.

20. Grissa O, Atègbo JM, Yessoufou A, et al. Antioxidant status and circulating lipids are altered in human gestational diabetes and macrosomia. Transl Res 2007;150:164-71.

21. Alexander BT. Placental insufficiency leads to development of hypertension in growth-restricted offspring. Hypertension 2003;41:457-62.

22. Woods LL, Ingelfinger JR, Nyengaard JR, Rasch R. Maternal protein restriction suppresses the newborn renin-angiotensin system and programs adult hypertension in rats. Pediatr Res 2001;49:460-7.

23. Woods LL, Weeks DA, Rasch R. Programming of adult blood pressure by maternal protein restriction: role of nephrogenesis. Kidney Int 2004;65:1339-48.

24. Gilbert JS, Nijland MJ. Sex differences in the developmental origins of hypertension and cardiorenal disease. Am J Physiol Regul Integr Comp Physiol 2008;295:R1941-52.

25. Lohmeier TE. The sympathetic nervous system and long-term blood pressure regulation. Am J Hypertens 2001;14(6 Pt 2):147S-54S.
26. Cavanal Mde F, Gomes GN, Forti AL, et al. The influence of L-arginine on blood pressure, vascular nitric oxide and renal morphometry in the offspring from diabetic mothers. Pediatr Res 2007;62:145-50.

27. MohanKumar SM, King A, Shin AC, Sirivelu MP, MohanKumar PS, Fink GD. Developmental programming of cardiovascular disorders: focus on hypertension. Rev Endocr Metab Disord 2007;8:115-25.

28. Nuyt AM, Alexander BT. Developmental programming and hypertension. Curr Opin Nephrol Hypertens 2009;18:144-52.

29. Segar JL, Roghair RD, Segar EM, Bailey MC, Scholz TD, Lamb FS. Early gestation dexamethasone alters baroreflex and vascular responses in newborn lambs before hypertension. Am J Physiol Regul Integr Comp Physiol 2006;291:R481-8.

30. Nehiri T, Duong Van Huyen JP, Viltard M, et al. Exposure to maternal diabetes induces salt-sensitive hypertension and impairs renal function in adult rat offspring. Diabetes 2008;57:2167-75.

31. Kinalski M, Sledziewski A, Telejko B, Zarzycki W, Kinalska I. Antioxidant therapy and streptozotocin-induced diabetes in pregnant rats. Acta Diabetol 1999;36:113-7.

32. Raza H, John A. Glutathione metabolism and oxidative stress in neonatal rat tissues from streptozotocin-induced diabetic mothers. Diabetes Metab Res Rev 2004;20:72-8.

33. Tarry-Adkins JL, Chen JH, Jones RH, Smith NH, Ozanne SE. Poor maternal nutrition leads to alterations in oxidative stress, antioxidant defense capacity, and markers of fibrosis in rat islets: potential underlying mechanisms for development of the diabetic phenotype in later life. FASEB J 2010;24:2762-71.

34. Shen JZ, Zheng XF, Kwan CY. Differential contractile actions of reactive oxygen species on rat aorta: selective activation of ATP receptor by $\mathrm{H} 2 \mathrm{O} 2$. Life Sci 2000;66:PL291-6.

35. Demirci B, McKeown PP, Dvm UB. The bimodal regulation of vascular function by superoxide anion: role of endothelium. BMB Rep 2008;41:223-9.

36. Chamiot-Clerc P, Renaud JF, Safar ME. Pulse pressure, aortic reactivity, and endothelium dysfunction in old hypertensive rats. Hypertension 2001;37:313-21.

37. Demirci B, McKeown PP, Bayraktutan U. Blockade of angiotensin II provides additional benefits in hypertension- and ageing-related cardiac and vascular dysfunctions beyond its blood pressure-lowering effects. J Hypertens 2005;23:2219-27.

38. Gomes P, Simão S, Silva E, et al. Aging increases oxidative stress and renal expression of oxidant and antioxidant enzymes that are associated with an increased trend in systolic blood pressure. Oxid Med Cell Longev 2009;2:138-45.

39. Silvia L, Miriam P, Petr K, Jaroslav K, Josef Z. Effects of aging and hypertension on the participation of endothelium-derived constricting factor (EDCF) in norepinephrine-induced contraction of rat femoral artery. Eur J Pharmacol 2011;667:265-70.

40. Butler DG, Butt DA, Puskas D, Oudit GY. Angiotensin II-mediated catecholamine release during the pressor response in rats. J Endocrinol 1994;142:19-28.

41. Gordon FJ, Mark AL. Impaired baroreflex control of vascular resistance in prehypertensive Dahl S rats. Am J Physiol 1983;245:H210-7.

42. Hashmi-Hill MP, Graves JE, Sandock K, Bates JN, Robertson TP, Lewis SJ. Hemodynamic responses elicited by systemic injections of flavin adenine dinucleotide in anesthetized rats. J Cardiovasc Pharmacol 2007;50:94-102.

43. Kober T, König I, Weber M, Kojda G. Diethyldithiocarbamate inhibits the catalytic activity of xanthine oxidase. FEBS Lett 2003;551:99-103.

44. Shastri S, Gopalakrishnan V, Poduri R, Di Wang H. Tempol selectively attenuates angiotensin II evoked vasoconstrictor responses in spontaneously hypertensive rats. J Hypertens 2002;20:1381-91.

45. Roghair RD, Miller FJ Jr, Scholz TD, Lamb FS, Segar JL. Endothelial superoxide production is altered in sheep programmed by early gestation dexamethasone exposure. Neonatology 2008;93:19-27. 